\title{
Channel, temporal, and composite uncertainty in the detection and recognition of auditory and visual signals*
}

\author{
D. C. EARLE and G. LOWE \\ University of Hull, Hull, England
}

In a $2 \mathrm{AFC}$ detection task, channel and temporal uncertainty were separately shown to produce decrements in the detectability of auditory and visual signals. The observed effect of channel uncertainty suggests that Ss are capable of using an "attentional" or "performance-compensation" strategy. This finding is discussed in relation to a "multiple-observation" model for channel uncertainty. Recognition measures were taken where appropriate and gave some support to the hypothesized attentional strategy. Under composite uncertainty conditions both types of uncertainty were presented simultaneously; the results indicated that any interaction effect was small, relative to the separate effects of channel and temporal uncertainty.

Most studies concerning the effects of stimulus uncertainty on the detection and recognition of signals have examined only a single dimension of uncertainty in each experiment. It would seem that, in detection situations outside the laboratory, Ss typically are required to detect signals that are unspecified with regard to more than one type of signal parameter, i.e., under conditions of composite uncertainty. The present experiment was designed to examine separately two dimensions of uncertainty, temporal and "channel", and to investigate the nature of the interaction (if any) between the different parameters in a detection and recognition task involving composite uncertainty.

Previous studies have shown that signal detectability decreases with short intervals of temporal uncertainty (ITU) up to about $4 \mathrm{sec}$, beyond which no further decrement is observed. This has been demonstrated for both auditory signals (Egan, Greenberg, \& Schulman, 1961) and visual signals (Lowe, 1967). With auditory detection the decrement at 4-sec ITU is equivalent to a reduction in signal energy of approximately $3 \mathrm{~dB}$.

Using a multiple-observation anaylsis, Kinchla (1969) has developed a general model and applied it specifically to temporal- and channel-uncertainty detection tasks. In the case of temporal uncertainty, it is assumed that the $S$ sums the information resulting from a series of observations. As the ITU increases relative to the duration of the signal, more observations of noise alone will be made, and this results in a decrement in detectability. In order to test the model, it is necessary to know the number of

*This rescarch was supported by a S.R.C. Research Studentship awarded to D.C.F: observations in each ITU. Kinchla, in fact, used up to six discrete observation intervals. He found that the S's ability to detect a tonal signal presented in a single randomly selected observation interval out of $n$ intervals decreased as $n$ increased, and was consistent with the model. The present experiment used a continuous period of temporal uncertainty (which, it is argued, is a more realistic uncertainty situation), as opposed to the discrete intervals of Kinchla's study. Consequently, Kinchla's model cannot be directly applied, since any artificial division of the continuous ITU into "discrete" observations would be entirely arbitrary.

The model can, however, be tested in the channel-uncertainty situation. Here it is assumed that the $S$ makes "simultaneous, independent and equally efficient observations in each channel." Information from the two observations is then summed, as with sequential observations. The model is contrasted with an "attentional" (i.e., "single filter") interpretation of the effects of channel uncertainty; it assumes that independent observations on each channel are integrated to produce a single decision. The present experiment provides a direct test of Kinchla's model for audio/visual channel uncertainty.

Very little work has been done specifically on audio/visual (A/V) uncertainty in a signal-detection situation. Kinchla (1969) deals with the problem only theoretically. Brown and Hopkins (1967), working in the framework of signal-detection theory (SDT) but using redundant signals, investigated the interaction between the auditory and visual modalities. They found evidence for a simple probabilistic summation of separate detection rates in the bisensory (A.V) condition, indicating perceptual independence. Eijkman and Vendrik
(1965) also presented bisensory A-V signals and observed that for amplitude discrimination the two channels appeared to be independent-Ss being capable of simultaneous and independent observations. The evidence from studies using redundant or bisensory signals is relevant to the case of channel uncertainty insofar as it supports a perceptual independence model for the auditory and visual modalities.

McGrath $(1962,1965)$ and Buckner and McGrath (1961), in a series of vigilance studies, used audio and visual signals in a number of different conditions. In one condition the two types of signals were given in separate sessions, and in another they were given together in the same session, but under nonredundant presentation, i.e., $A / V$ uncertainty. They found a "performance sharing" effect in the channel-uncertainty condition: that is, performance deteriorated on the weaker signal and improved with the stronger signal, irrespective of which modality contained the stronger signal. It was not possible from McGrath's analysis to determine whether or not there was a significant overall decrement in detectability due to $A / V$ uncertainty, since detection measures (as in most vigilance studies) were based on hit rates (HRs). There was, however, a trend toward more false alarms (FARs) in the A/V condition in all cases, even though the observed FARs were very low. Using SDT analysis, some studies have demonstrated a decrement in performance due to channel uncertainty (e.g., Lowe \& Earle, 1968).

Recognition responses may also be made in the $A / V$ uncertainty condition. Egan and Benson (1966) have shown that such additional responses on the part of the $S$ do not interfere with the detection task. If attentional factors are involved in the channel-uncertainty situation, then recognition responses should give some indication of this. For example, if the $S$ were attending more to one type of signal, it might be expected that recognition performance would be better for this signal. No specific predictions can be made from a multiple-observation model for recognition performance; the general expectation here would be that recognition should be directly related to signal detectability.

With regard to the compositeuncertainty situation, it is important to determine how the uncertainty parameters interact. One possibility is that of a simple summation of the separate effects resulting from each dimension of uncertainty (i.e., no obvious interaction). Such a hypothesis has been used to predict decrements in detectability under composite uncertainty 
in a previous study involving lateral and temporal uncertainty (Lowe \& Earle, 1969). The three Ss in that study (of auditory detection) showed somewhat different patterns of results, and the summation hypothesis was not found to be a very good predictor. This seemed to indicate that there may have been some interaction, but that it could well be complex and may depend considerably on the detection strategy of the individual $S$. In another study involving lateral (L/R) and $A / V$ uncertainty (Lowe \& Earle, 1968), the summation hypothesis was found to underpredict in the composite condition.

Neither of these two studies shed very much light on the composite-uncertaintydetection task, and the present investigation was designed as a further examination of this problem. In addition, the predictions of Kinchla's multiple observation model are examined in relation to the $\mathrm{A} / \mathrm{V}$ channel-uncertainty situation.

\section{METHOD}

The experimental situation was a two-alternative, forced-choice (2AFC) detection task with two intervals. In one set of conditions the signal was specified auditory, with various degrees of temporal uncertainty-ITU $=0,2,4 \mathrm{sec}$ (and $8 \mathrm{sec}$ for $S 1$ ); in another set the signal was specified visual, again with the same ITUs. A third set of conditions included both auditory and visual signals with $A / V$ uncertainty, as well as the periods of temporal uncertainty. In these latter conditions a recognition response (A or $V$ ) was also required.

Signals were presented in the auditory and visual modalities. The auditory signal was an 85 -msec square-wave pulse of $1,000 \mathrm{~Hz}$ fed dichotically into a set of balanced earphones (Akai, 8-ohm) and masked by white noise from a Dawes WN generator. The visual signal was an increment in the brightness of an electroluminescent panel $(2 \times 2$ in; $1-\mathrm{kc}$ operation, up to $250 \mathrm{~V}$ ). This brightness increment was produced by an $85-\mathrm{msec}$ square-wave pulse of $500 \mathrm{~Hz}$ from a multifrequency oscillator. The visual panel was set into a viewing piece that excluded all other illumination during observation; viewing distance (from eye to panel) was about $21 \mathrm{~cm}$. Testing took place in a light-proof room illuminated only by a 15-W bulb.

For all three Ss the level of WN was $22.5 \mathrm{mV}$ measured at the input to the ea rphones (approximately $72 \mathrm{~dB}$ re 0.0002 microbar, with a bandwidth of $20 \mathrm{Kc} / \mathrm{sec})$. The background illumination of the panel was $11.5 \mathrm{~V}$. Signal intensities for each $S$ were held constant at an

Table 1

Detection Performance Under Conditions of Channel Uncertainty (A/V UNC) and No Uncertainty (SS). Probability values indicate significance levels based on the difference between proportions of correct detections under the specified and uncertainty conditions.

\begin{tabular}{llcccccc} 
& \multicolumn{3}{c}{ Auditory } & & \multicolumn{3}{c}{ Visual } \\
\cline { 2 - 4 } & d'SS & $\mathrm{d}^{\prime}$ A/V UNC & $\mathrm{p}$ & & $\mathrm{d}^{\prime}$ SS & $\mathrm{d}^{\prime}$ A/V UNC & $\mathrm{p}$ \\
\hline S1 & 2.78 & 1.98 & .025 & 2.03 & 2.03 & n.s. \\
S2 & 3.28 & 2.40 & .025 & 1.81 & 1.78 & n.s. \\
S3 & 1.07 & 0.69 & .025 & 1.63 & 1.12 & $<.01$ \\
\hline
\end{tabular}

appropriate level, as determined by preliminary testing using about 200 trials for both the auditory and visual signals. For $S 1$ the auditory signal was $15.0 \mathrm{mV}$ added to the WN, measured at input to the earphones, and the visual signal was an increment of $100 \mathrm{mV}$ added to the background intensity. In the case of Ss 2 and 3 , the signal levels were, respectively: $17.0 \mathrm{mV}$ (auditory) and $175 \mathrm{mV}$ (visual), and $13.5 \mathrm{mV}$ (approximately $52 \mathrm{~dB}$ ) and $120 \mathrm{mV}$ (visual).

Observation intervals and ITUs were indicated by a finger vibrator on the S's left hand. The presentation time of a signal within an ITU was randomized. A trial began with a warning signal of $1 \mathrm{sec}$ duration, followed by a 1-sec pause and then the two observation intervals (the duration of which was determined by the ITU) separated by $1 \mathrm{sec}$. The $S$ recorded his responses during a $4-\mathrm{sec}$ intertrial interval.

Trials were given in blocks of 20 in all conditions. The same ITU was used throughout any given block of trials. At the beginning of each block of trials, the relevant signals were presented several times in order to familiarize the $S$, and at the beginning of each session 20 practice trials with both $\mathrm{A}$ and $\mathrm{V}$ signals were given. There was a short rest pause after every block, and a longer one halfway through the session. During these breaks, the WN was attenuated. Both $S 2$ and $S 3$ had 10 sessions, each lasting about $75 \mathrm{~min}$ and containing 240 trials; S 1 had eight sessions, each lasting about $90 \mathrm{~min}$ and containing 320 trials. All conditions were presented in a randomized order, in any given session.

Three Ss were run separately. One $S$ (S 1) was one of the authors (DCE), the other two were graduate students. All Ss were fully aware of the relevant probabilities in the various conditions of the experiment; none of them were given any feedback during the experimental sessions.

A SDT analysis was used to estimate the performance parameters under the different conditions. $P($ correct) values were obtained from the data, in order to determine $d^{\prime}$ (detectability index) and $d_{R}^{\prime}$ (recognition parameter, cf. Tanner, 1956).

\section{RESULTS}

Table 1 shows the main effect on signal detectability of $A / V$ channel uncertainty. As the experimental situation was a $2 A F C$ paradigm, tests of significance ( $\mathrm{z}$ test) were carried out on the relevant proportions- $P($ correct $)$. The significance levels are indicated in the table.

With Ss 1 and 2 there were significant decrements for the stronger auditory signals and no effect for the less detectable visual signals. In the case of S 3, with much lower detectabilities and with better performance on the visual than on the auditory signals, there was a different pattern of results. Both auditory and visual signals were significantly less detectable in the channel-uncertainty conditions.

Predicted detection performance for the A/V uncertainty condition was derived from Kinchla's model, whereby $\mathrm{d}_{\mathrm{AV}}^{\prime}=$ $0.5\left(2^{-1 / 2} \mathrm{~d}_{\mathrm{A}}^{\prime}+2^{-1 / 2} \mathrm{~d}_{\mathrm{V}}^{\prime}\right)$, where 2 refers to the number of alternative signal channels and $d^{\prime}{ }_{A}$ and $d^{\prime}{ }_{V}$ are the observed detectabilities in the signal specified (SS) conditions. These predicted and observed $\mathrm{d}^{2}$ vallues are presented in Table 2 . Overall, the comparisons give some support to Kinchla's model, although Ss 1 and 2 performed somewhat better than predicted.

Table 3 shows the effects of temporal uncertainty. In these conditions the signal was specified (A or V), and there were various ITUs. All Ss showed highly significant decrements in $\mathrm{d}^{\prime}$ with temporal uncertainty, for both auditory and visual signals.

Observed detection performance in the composite uncertainty conditions, together with predicted performance based on Kinchla's model, is shown in Table 4. Observed detectabilities in the temporal uncertainty conditions with specified

Table 2

Comparison of Observed Detection Performance Under Conditions of Channel Uncertainty With Predicted Performance Based on Kinchla's Model ( $d$ ' Based on $A$ and $V$ Signals Combined.)

\begin{tabular}{llll} 
& \multicolumn{2}{c}{$\mathrm{d}^{\prime} \mathrm{A} / \mathrm{V}$ UNC } & \\
\cline { 2 - 3 } & Obs. & Pred. & $\mathrm{p}$ \\
\hline S1 & 2.00 & 1.70 & .057 \\
S2 & 2.03 & 1.80 & n.s. \\
S3 & 0.88 & 0.96 & n.s. \\
\hline
\end{tabular}


Table 3

Detection Performance Under Conditions of Temporal Uncertainty (ITU $=2,4$, or $8 \mathrm{Sec}$ ) and No Temporal Uncertainty (ITU-0) for Auditory and Visual Signals. Entries indicate values of $d^{\prime}$.

\begin{tabular}{llllllllll}
\hline & \multicolumn{3}{c}{ Auditory } & & \multicolumn{3}{c}{ Visual } \\
\cline { 2 - 5 } & ITU-0 ITU-2 ITU-4 ITU-8 & & ITU-0 ITU-2 ITU-4 ITU-8 \\
\hline S1 & 2.78 & 1.42 & 1.35 & 1.05 & & 2.03 & 1.55 & 0.92 & 0.89 \\
S2 & 3.28 & 1.70 & 1.56 & $-\ldots$ & & 1.81 & 1.00 & 0.95 & -- \\
S3 & 1.07 & 0.30 & 0.47 & -- & & 1.63 & 1.14 & 0.60 & - \\
\hline
\end{tabular}

Table 4

Comparison of Observed Detection Performance Under Conditions of Composite Uncertainty (A/V Uncertainty Together With Temporal Uncertainty) With Predicted Performance Based on Kinchla's Model.

\begin{tabular}{|c|c|c|c|c|c|c|c|c|c|}
\hline & \multicolumn{3}{|c|}{$\begin{array}{l}\text { ITU-2 } \\
\mathrm{d}^{\prime} \mathrm{A} / \mathrm{V}\end{array}$} & \multicolumn{3}{|c|}{$\begin{array}{l}\text { ITU-4 } \\
\mathrm{d}_{\mathrm{A} / \mathrm{V}}\end{array}$} & \multicolumn{3}{|c|}{$\begin{array}{l}\text { ITU-8 } \\
\mathrm{d}^{\prime} \mathrm{A} / \mathrm{V}\end{array}$} \\
\hline & Obs. & Pred. & $p$ & Obs. & Pred. & $\mathrm{p}$ & Obs. & Pred. & $\mathrm{p}$ \\
\hline S1 & 1.01 & 1.05 & n.s. & 0.94 & 0.80 & n.s. & 0.91 & 0.69 & $<.06$ \\
\hline S2 & 1.13 & 0.96 & n.s. & 0.84 & 0.89 & n.s. & -- & -- & \\
\hline S3 & 0.46 & 0.51 & n.s. & 0.44 & 0.38 & n.s. & -- & - & \\
\hline
\end{tabular}
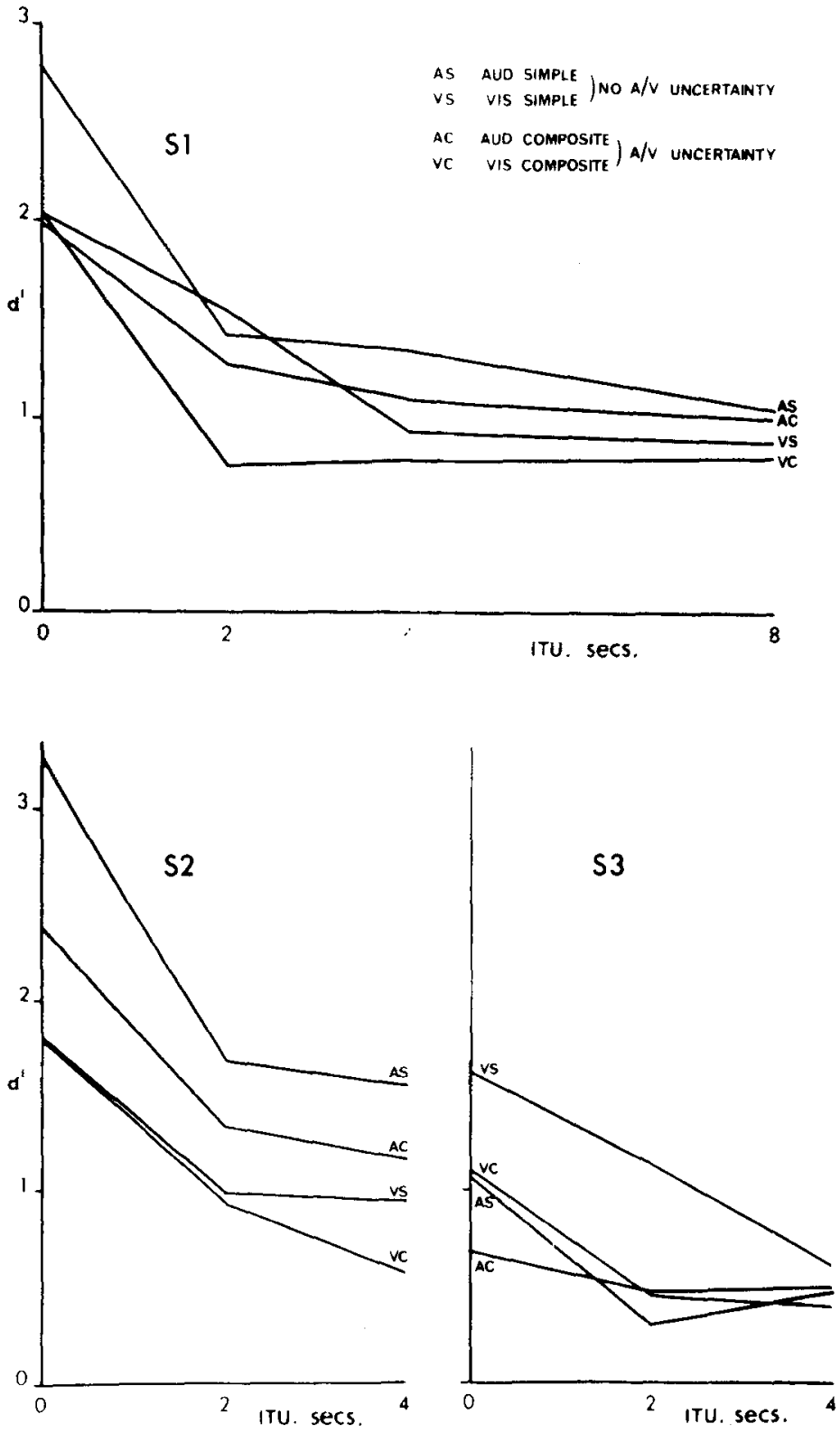

Fig. 1. Detectability $\left(d^{\prime}\right)$ as a function of ITU. For $S$ 1, each point was based on 160 trials; for $S 2$ and $S 3$, there were 200 trials. signals were used in the derivation of predicted $d^{\prime}{ }_{A} \dot{V}$ values for the sarne ITUs.

There were no significant differences between the observed and predicted values. Kinchla's model seems to provide a reasonably close fit to the data in the $A / V$ channel-uncertainty condition, whether or not there is also temporal uncertainty.

The data were then examined for each signal separately (Fig. 1). It can be seen that Ss 1 and 2, whose performance was not affected by channel uncertainty for the visual signals, now showed significant decrements in visual detectability under composite uncertainty for ITU-2 (S 1) and ITU-4 (S 2).

Figure 2 shows $d_{R}^{\prime}$, the recognition parameter (Tanner, 1956), compared with $\mathrm{d}^{\prime} \mathrm{AV}$, and $\mathrm{P}$ (correct recognition) for the auditory and visual signals. The change in $d_{R}^{\prime}$ with ITU closely follows that of $d^{\prime}{ }_{A V}$. In all cases, values of $P(C R)$ for the auditory and visual signals were very nearly equal with ITU-0 and ITU.2. However, with ITU-4 there is some divergence between $\mathrm{A}$ and $\mathrm{V}$ recognition, which does not appear to relate consistently to the relative detectabilities of the two signals.

For all three Ss no significant differences were found between the detection rates for signals in the first and second halves of the ITUs or in the first and second observation intervals.

\section{DISCUSSION}

It seems clear that temporal uncertainty reduces the detectability of auditory and visual signals under both simple and composite uncertainty conditions. This effect gives an increasing decrement with auditory signals, at least up to ITUs of $2 \mathrm{sec}$ and in some cases with visual signals up to ITUs of $4 \mathrm{sec}$. This is consistent with the findings of Egan et al (1961) for auditory signals and Lowe (1967) for visual signals. It is interesting to note that even with signals of relatively low detectability (e.g., in the case of S 3) there was still a significant effect of temporal uncertainty.

The case of $\mathrm{A} / \mathrm{V}$ channel uncertainty is more complex. In the simple (ITU-0) condition, all three $\mathrm{Ss}$ showed a performance decrement, although with $S 1$ and $S 2$ this was confined to the more detectable auditory signals. McGrath 

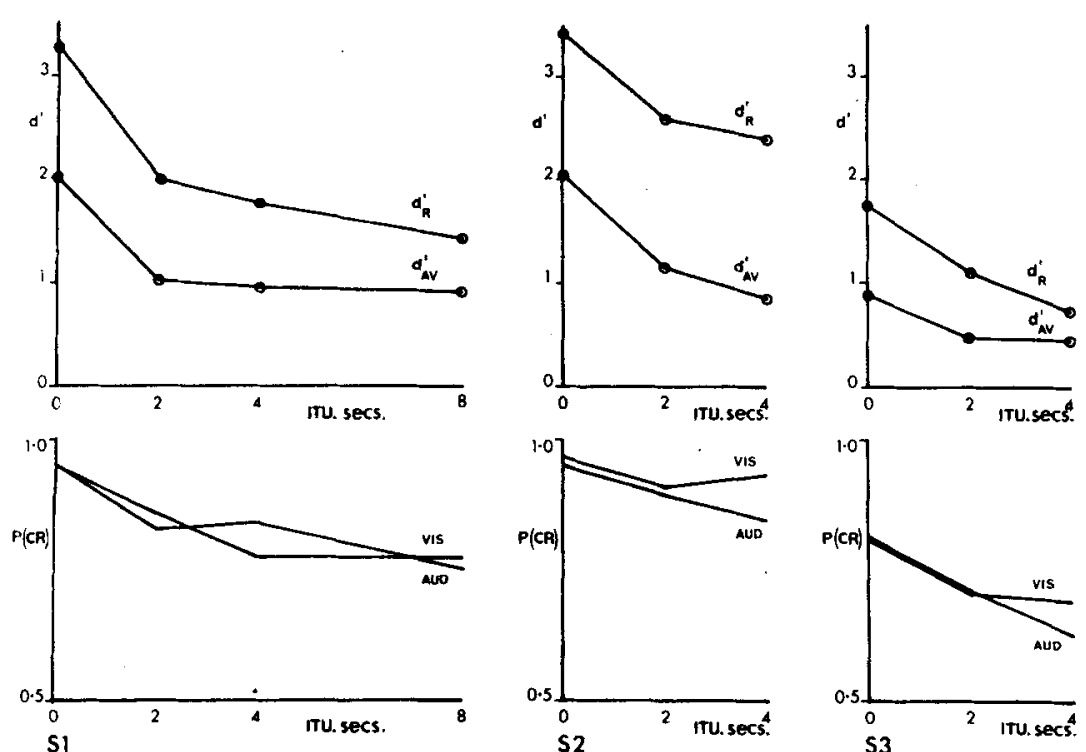

One of the difficulties in investigating the effects of composite uncertainty is the problem of how to interpret the data. The present authors (Lowe \& Earle, 1968, 1969) have previously used a summation hypothesis. The disadvantage of this is that it relies on a single estimate of one dimension of uncertainty for the predictions. For example, in the present situation the effects of channel uncertainty would be estimated from only the ITU-0 condition. However, by hypothesizing that the effects of channel uncertainty are independent of those of temporal uncertainty, Kinchla's model for $\mathrm{A} / \mathrm{V}$ uncertainty can be used to predict the $\mathrm{d}^{\prime}$ values in the composite conditions and thus test the hypothesis. The results of this test gave some support to the notion that the two dimensions of uncertainty are independent (Table 3). This conclusion only holds, of course, if Kinchla's model is a good predictor in the simple $A / V$ uncertainty situation. Overall support was found for the model, but it appeared that Ss could improve their performance by using an attentional strategy. If the auditory and visual data for the composite conditions are considered separately, then it does appear that the introduction of temporal uncertainty can, under some circumstances, have an effect on channel uncertainty. Ss 1 and 2, whose performance was not affected by channel uncertainty on the visual signals, failed to maintain this performance throughout the ITU range. This could be interpreted simply as a change on the part of the Os from an attentional to a nonattentional strategy. Alternatively, it could be some slight interaction effect between the two dimensions of uncertainty. However, from the evidence available at present, it must be concluded that any such interaction effect is rather small, and that the composite uncertainty situation can best be described by treating the two types of uncertainty separately with reference to the kinds of strategies used by the Ss.

\section{REFERENCES}

BROWN, A. E., \& HOPKINS, H. K. Interaction of the auditory and visual sensory modalities. Journal of the Acoustical Socicty of America, $1967,41,1.6$.

BUCKNER, D. N., \&. MorRATH, J. J. A comparison of performances on single and dual sensory mode vigilance tasks. Technical Report ASW No. 8, Human liactors Rescarch, Inc., Los Angeles, 1961.

IEGAN, J. P., \& BENSON, W. Lateralization of a weak signal presented with correlated and with uncorrclated noise. Journal of the Acoustical Socicty of America, 1966, 40, 20-26.

EGAN, J. P., GREENBERG, G. Z., \& SCHULMAN, A. I. Interval of time uncertainty in auditory detection. Journal of the Acoustical Society of America, 1961, 33, 771-778. 
EIJKMAN, E., \& VENDRIK, A. J. H. Can a sensory system be specified by its internal noise? Journal of the Acoustical Society of America, 1965, 37, 1102-1109.

KINCHLA, R. A. Temporal and channel uncertainty in detection: A multiple observation analysis. Perception \& Psychophysics, 1969, 5, 129-136.

LOWE, G. Interval of time uncertainty in visual detection. Perception \& Psychophysics, 1967 2, 278-280.

LOWE, G., \& EARLE, D. C. Auditory detection and recognition under conditions of simple and composite uncertainty. Paper presented to the British Psychological Society, London Conference, December 1968.

LOWE, G., \& EARLE, D. C. Auditory detection and recognition under conditions of lateral, temporal, and composite uncertainty. Journal of the Acoustical Society of America, 1969, 45, 1485-1488.

McGRATH, J. J. Performance sharing in dual-mode monitoring. HFR Technical Report No. 740-1, Human Factors Research, Inc., Los
Angeles, 1962

McGRATH, J. J. Performance sharing in an audio-visual vigilance task. Human Factors, 1965, 7, 141-153.

MORAY, N. Attention: Selective processes in vision and hearing. London: Hutchinson, 1969.

TANNER, W. P., JR. Theory of recognition. Journal of the Acoustical Society of America, $1956,28,882-888$.

(Accepted for publication July 6, 1970.) 